\title{
Coding of coronary arterial origin and branching in congenital heart disease: The modified Leiden Convention
}

Adriana C. Gittenberger-de Groot, PhD, ${ }^{a}$ Wilke M. C. Koenraadt, MD, ${ }^{a}$ Margot M. Bartelings, MD, PhD, ${ }^{b}$ Regina Bökenkamp, MD, PhD,${ }^{\mathrm{c}}$ Marco C. DeRuiter, PhD, ${ }^{\mathrm{b}}$ Mark G. Hazekamp, MD, PhD, ${ }^{\mathrm{d}}$ Ad J. J. C. Bogers, MD, PhD, ${ }^{e}$ Jan M. Quaegebeur, MD, PhD, ${ }^{f}$ Martin J. Schalij, MD, PhD, ${ }^{a}$ Hubert W. Vliegen, MD, PhD, ${ }^{a}$ Robert E. Poelmann, PhD, ${ }^{a, g}$ and Monique R. M. Jongbloed, MD, PhD ${ }^{a, b}$

\section{ABSTRACT}

Objectives: Variations in coronary anatomy are common and may relate to the position of the coronary ostium relative to the aortic sinus, the angle of coronary take-off, or the course of the coronary arterial branches. Several classification systems have been proposed. However, they all lack a simple rationale that is applicable irrespective of the relative position of the great arteries, as well as in bicuspid aortic valves. We present a modification of a relatively simple system introduced in the early 1980s, designated the "Leiden Convention."

Methods: The first step of the Leiden Convention is that the clinician takes position in the nonfacing sinus of the aorta looking toward the pulmonary orifice. The right-hand facing sinus is sinus 1 , and the left-hand facing sinus is sinus 2 . The coronary branches arising from sinus 1 are annotated proceeding in a counterclockwise fashion toward sinus 2. "Usual" (normal) coronary anatomy would be 1R-2LCx. Given their clinical relevance, single sinus coronary arteries are discussed separately.

Results: This system was originally designed and highly applicable in hearts with an altered great artery relationship, such as in the variable and complicated patterns seen in transposition of the great arteries and double outlet right ventricle. The modified system also can be used in cases with normally related great arteries, cases with single sinus coronary arteries, and cases with bicuspid aortic valves.

Conclusions: The modified Leiden Convention is not a strict classification but a simple coronary coding system that is broadly applicable. (J Thorac Cardiovasc Surg 2018;156:2260-9)

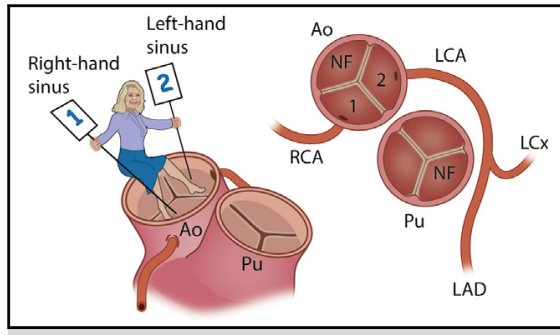

The modified Leiden Convention coronary coding system.

\section{Central Message}

The Leiden Convention is a coding system that is applicable in hearts with variable and complicated coronary patterns irrespective of the position of the great arteries.

\section{Perspective}

The Leiden convention can be applied in cases with CHD, including BAVs, as well as in structurally normal hearts. Single sinus coronary arteries are included and discussed as a separate group.

See Editorial Commentary page 2270.
Coronary arterial origin and course of the proximal main stems can be important indications for analysis of cardiac patho-morphology. In the morphologically normal heart,

\footnotetext{
From ${ }^{a}$ Cardiology, Leiden University Medical Center, Leiden, The Netherlands; bAnatomy and Embryology, Leiden University Medical Center, Leiden, The Netherlands; ${ }^{\mathrm{c}}$ Pediatric Cardiology, Leiden University Medical Center, Leiden, The Netherlands; ${ }^{\mathrm{d}}$ Cardiothoracic Surgery, Leiden University Medical Center, Leiden, The Netherlands; ${ }^{e}$ Cardiothoracic Surgery, Erasmus Medical Center, Rotterdam, The Netherlands; ${ }^{\mathrm{f}}$ Pediatric Cardiac Surgery, Presbyterian Morgan Stanley Children's Hospital, New York, NY; and ${ }^{\mathrm{g}}$ Department of Animal Sciences and Health, Leiden University, Leiden, The Netherlands.

Received for publication April 13, 2018; revisions received July 15, 2018; accepted for publication Aug 2, 2018; available ahead of print Sept 19, 2018.

Address for reprints: Adriana C. Gittenberger-de Groot, PhD, Department of Cardiology, Leiden University Medical Center, Postal Zone S-1-P, PO Box 9600, 2300 RC Leiden, The Netherlands (E-mail: acgitten@lumc.nl).

0022-5223

Copyright (C) 2018 by The American Association for Thoracic Surgery. This is an open access article under the CC BY-NC-ND license (http://creativecommons.org/ licenses/by-nc-nd/4.0/).

https://doi.org/10.1016/j.jtcvs.2018.08.009
}

there is a fairly consistent coronary pattern consisting of 3 main branches connected by way of 2 orifices to the semilunar sinuses of the aorta. The left coronary artery (LCA) arises from the left sinus that, taking a curving course posterior to the pulmonary orifice, divides into a left anterior descending (LAD) and a left circumflex (LCx) branch. These branches run subepicardially in the anterior interventricular sulcus and the left atrioventricular sulcus, respectively. The right coronary artery (RCA) connects to the right sinus and runs in the right atrioventricular sulcus.

Scanning this QR code will take you to the article title page. 

Abbreviations and Acronyms
$\mathrm{BAV}=$ bicuspid aortic valve
CHD = congenital heart disease
$\mathrm{DORV}=$ double outlet right ventricle
LAD $=$ left anterior descending
LCA = left coronary artery
LCx $=$ left circumflex
$\mathrm{RCA}=$ right coronary artery
SCD = sudden cardiac death
TGA $=$ transposition of the great arteries

From the LAD, LCx, and RCA, several marginal, septal, and diagonal branches arise that enter the myocardium and perfuse the septum and free wall of the ventricles. Smaller separate branches are important for perfusion of the sinoatrial and atrioventricular nodes. They usually originate from a main branch that is closest to the respective node, although diversity in branching patterns is found. ${ }^{2,3}$ As a general rule, coronary arteries will never cross over other coronary artery branches in their course.

\section{MATERIALS AND METHODS}

\section{Variations in Sinus Terminology as Related to Position of the Great Arteries}

Variations in coronary anatomy are commonly encountered and may relate to the position of the coronary ostium in the aortic sinus, the angle of the coronary take-off, and the course of the coronary arterial branches.
Specifically in case of congenital heart disease (CHD) involving the cardiac outflow tract, variations are seen in origin and branching patterns. ${ }^{1}$ This is even more so when there are variations in the relative position of the orifices of the great arteries. In the normal situation, the aortic orifice is located right posterior with regard to the pulmonary orifice. In cases of CHD affecting the outflow tract, the position of the aorta may vary from right posterior, to right side-by-side, to right anterior, to frontal, or to left anterior as related to the pulmonary orifice (Figure $1, A$ ). When evaluating the position of the aortic valve leaflets with regard to the pulmonary valve, regardless of the position, 2 sinuses can be recognized facing the pulmonary valve (referred to as "facing" sinuses) and 1 "nonfacing" sinus (Figure 1, A:I and $B: I$ ). In the aorta, the nonfacing sinus is often referred to as the "noncoronary sinus."

To enable the proposed simple unambiguous coding, first a terminology for the aortic sinuses was adopted that is independent of the relative anatomic position of the great arteries and the position of the aortic orifice with regard to the anterior surface of the heart. In this way, it has been possible to avoid the change in positional nomenclature for the semilunar sinuses when the aorta is not in its normal position with regard to the pulmonary orifice, as is the case in transposition of the great arteries (TGA), congenitally corrected transposition, and double outlet right ventricle (DORV). With a positional terminology for the semilunar sinuses, this may lead to a myriad of variations (Figure 1, AII-V). The terminology for the semilunar sinuses in the Leiden Convention is independent of the position of the great arteries (Figure 1,BII-V). This is supported by cardiac development, when the endocardial outflow tract cushions, present in the initially unseparated outflow tract, become involved in septation. Neural crest-derived cells form the so-called condensed mesenchyme separating the future aortic and pulmonary orifices (Figure 2, $A-D$ ). At the level of the orifices, 2 facing commissures are formed, 1 in the aortic and 1 in the pulmonary orifice (Figure 2, B and $C$ ). At both opposite sides, 2 more commissures are formed between the intercalated (future nonfacing leaflets) and the main endocardial outflow tract cushions (facing leaflets) (Figure 2, $B$ and $C$ ). This eventually results in the definitive aortic and
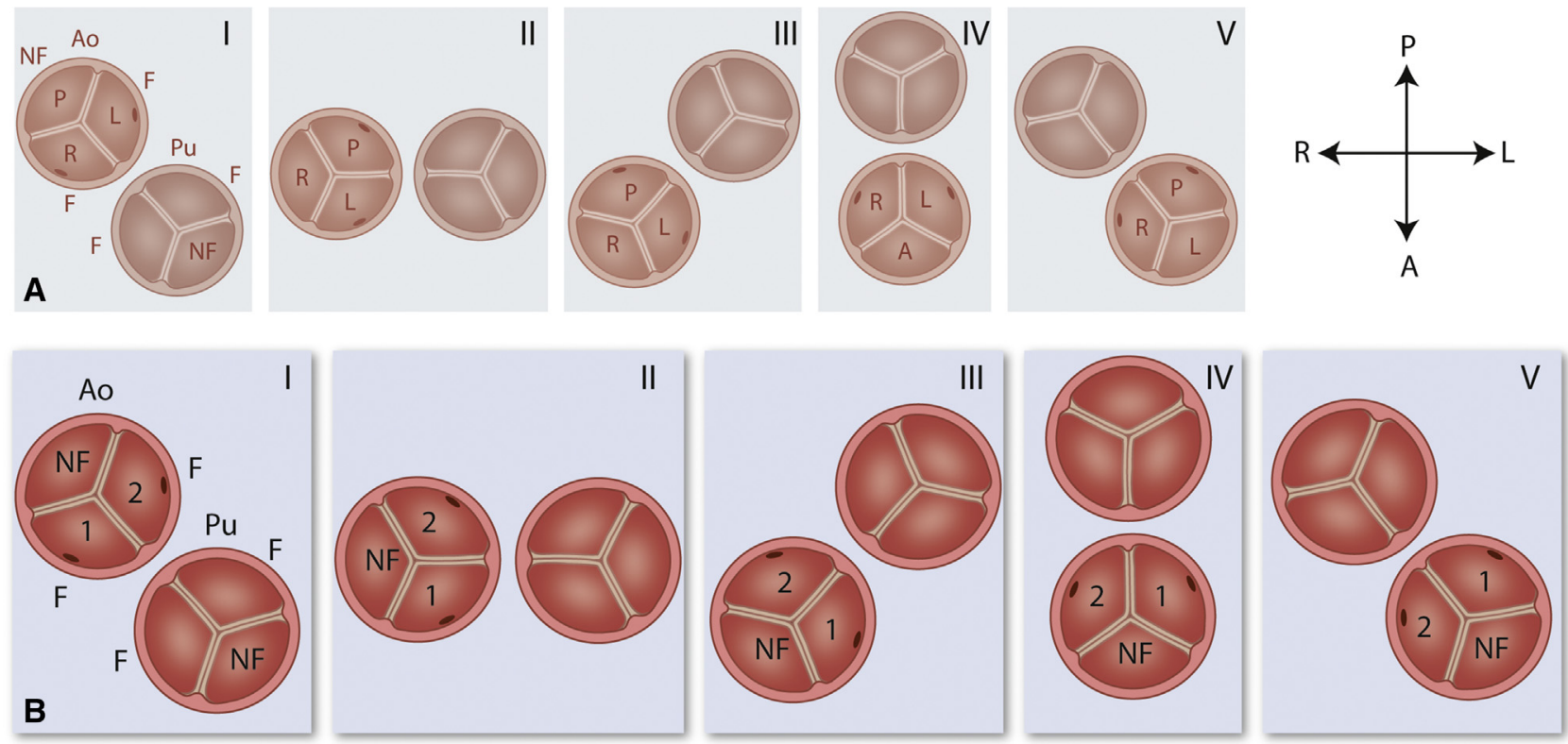

FIGURE 1. View from above. A, Influence of positional changes of the great arteries on the description of the semilunar sinuses. Regardless of the position of the aortic valve, 2 sinuses can be recognized facing the pulmonary valve. These are referred to as the facing sinuses, leaving 1 nonfacing sinus. B, Unambiguous numeric terminology that is not influenced by relative position of the great arteries: $P$, Posterior; $R$, right; $L$, Left; $A$, Anterior. Relative position of the aortic orifice: $\mathrm{I}=$ right posterior, $\mathrm{II}=$ right side-by-side, $\mathrm{III}=$ right anterior, $\mathrm{IV}=$ anterior, $\mathrm{V}=$ left anterior. $A o$, Aortic; $N F$, nonfacing; $F$, facing; $P u$, pulmonary. 


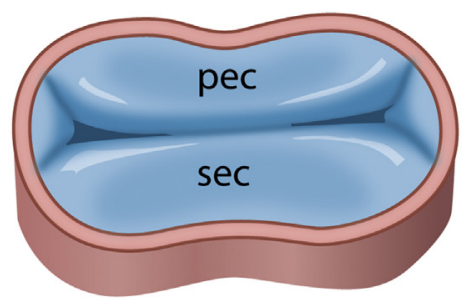

A

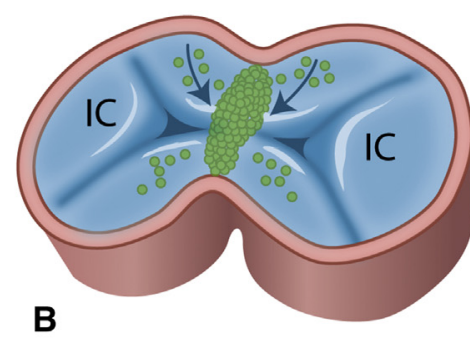

B

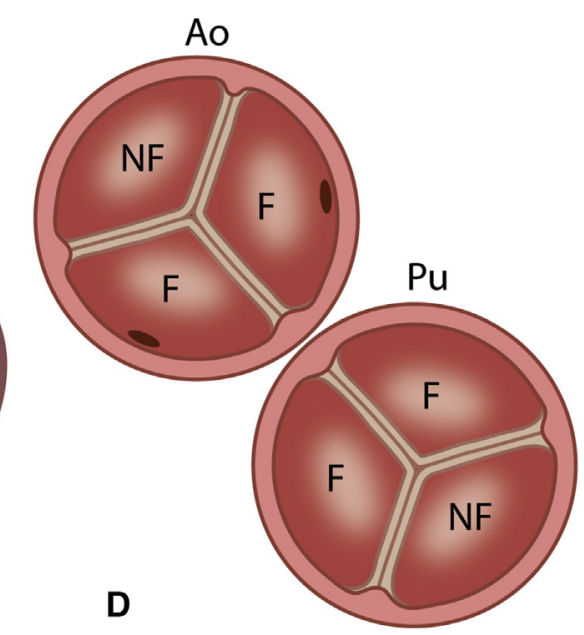

FIGURE 2. View from above. A, Unseptated cardiac outflow tract at the future orifice level, showing a large septal and parietal outflow tract endocardial cushion and 2 small intercalated cushions. B, Septation of the orifice level with ingrowth of neural crest cells (green), in which 2 facing commissures (arrows) are formed between the aortic and the pulmonary orifice (B, C). D, The definitive situation in which each orifice contains 2 facing and 1 nonfacing sinus. pec, Parietal; sec, septal; $I C$, intercalated cushion; $N F$, nonfacing; $F$, facing; $A O$, aortic; $P u$, pulmonary.

pulmonary valves (Figure 2, $C$ and $D$ ) with their typical position of the commissures, including 2 facing commissures.

The general rule holds that the mentioned main coronary branches take the shortest course toward an aortic semilunar sinus. This finding is supported by the developmental background showing that the coronary arteries grow into the aortic sinuses instead of growing out. ${ }^{5,6}$ There is a still an unexplained preference for the facing sinuses ( $99 \%$ of the cases), leaving the nonfacing sinus mostly without an orifice of a connecting branch (noncoronary sinus). Over time, molecular research in mainly mouse models attempted to elucidate this phenomenon. A recent publication is enlightening in this respect, because it shows a separate developmental program guiding the LCA and RCA, however, without explaining the preference for the facing sinuses of the aorta and the avoidance of the nonfacing/noncoronary sinus. ${ }^{7}$

With the advance of corrective surgery for $\mathrm{CHD}$ and the introduction of sophisticated diagnostic imaging techniques over the past decades, it has become possible to document the coronary artery anatomy before corrective or palliative surgery is performed. Specifically, the arterial switch procedure for TGA and DORV with subpulmonary ventricular septal defect (Taussig-Bing malformation), requiring an additional transfer of the coronary arterial ostia into the neoaorta, triggered an increased interest in variations in coronary artery anatomy. ${ }^{8}$

A special group of coronary malformations concern the coronary arteries arising from 1 coronary sinus. These are referred to as "single sinus coronary arteries," irrespective of whether there is only 1 shared coronary ostium or more ostia lying close together in the same coronary sinus. Single sinus coronary arteries can exist isolated or in the setting of other congenital malformations. In contrast to the common variations encountered in coronary anatomy, single sinus coronary arteries seem to be more clinically relevant, especially when there is an interarterial/intramural course of a coronary branch..$^{9-11}$ Preoperative information is also of relevance in patients with bicuspid aortic valves (BAVs).

\section{Principles of the Coronary Artery Coding System: The Leiden Convention}

Several classification systems have been proposed in the last 30 years, ${ }^{12}$ all lacking, however, a simple nonconfusing rationale that is applicable irrespective of the position of the great arteries, as well as in situations in which only 2 valve leaflets are present as in BAV. In the early 1980s, we provided a relatively simple coding system ${ }^{4}$ that was later on dubbed the "Leiden Convention." 8 However, this system has a few flaws, mainly because it does not adequately show the course of the coronary branches (curving) around the great arteries. A modification to this system was proposed $^{13}$ but never made it to referenced literature. The modified Leiden Convention, as is presented in this article, is applicable in hearts with an altered great artery relationship such as encountered in TGA and DORV, presenting with variable and complicated patterns, as well as in cases with normally related great arteries, including BAV. Given their clinical relevance, single sinus coronary arteries will be discussed as a separate group.

For application of the Leiden Convention, as a general rule, an aortic valve with 3 leaflets always carries a nonfacing/noncoronary sinus. The first step of the Leiden Convention is that the clinician or researcher takes position in the nonfacing sinus of the aorta looking toward the pulmonary orifice and then referring to the right-hand facing sinus as sinus 1 and to the left-hand facing sinus as sinus 2 (Figure 3, A). Independent of the position of the great arteries, this relationship is always maintained, thus acquiring the desired simplification of semilunar sinus terminology (Figure 1,B). 


\section{Step 1}

Position in the non-facing sinus

- Right-hand sinus: sinus 1

- Left-hand sinus: sinus 2
Step 2

Annotation coronary arteries

- Start with sinus 1

- Name encountered branches

in counter clockwise order

- $\mathrm{L}=$ left anterior descending coronary artery (LAD)

- Cx = left circumflex artery (LCX)

Step 3

Variations in coronary orifice origin

- Branches originating from a separate

sinus are separated by a dash

- Separate orginating branches

are interposed by a comma

- Branches derived from one stem

have no comma

- Interarterial course is indicated

by an asterisk
- $\mathrm{R}=$ right coronary artery
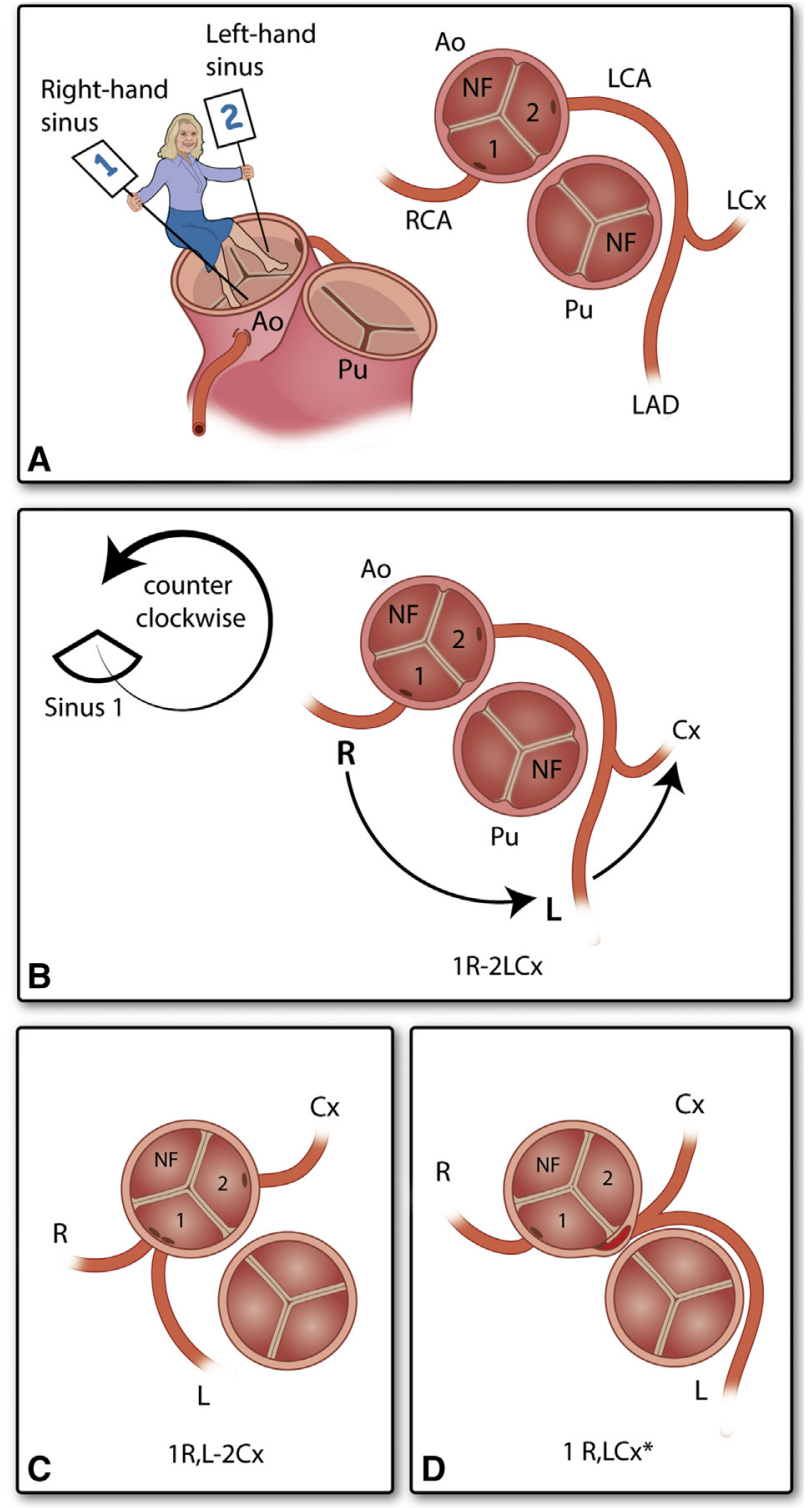

Associated characteristics with respect to coronary course/anatomy

- Interarterial/intramural course

- Relative relationship of the great arterial orifices

- Acute angle (vertical/horizontal)

of coronary artery

- Degree of alignment of facing commisures

- Eccentric position of coronary orifices in horizontal/vertical plane

- Single, common, double orifices

- Accessory branches

- Left-right dominance

- Type B BAV with inconclusive morphology: describe coronary anatomy extensively

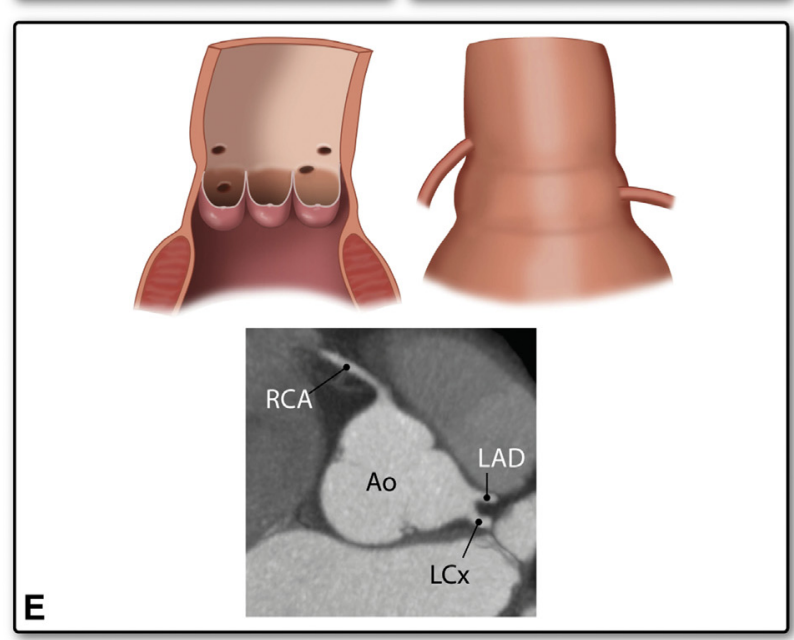

FIGURE 3. Application of the Leiden Convention applying 3 subsequent steps (A-D) and additional information (E). For explanation, see text. Ao, Aortic; $N F$, nonfacing; $L C A$, left coronary artery; $R C A$, right coronary artery; $L C x$, left circumflex; $P u$, pulmonary; $L A D$, left anterior descending; $R$, right; $C x$, circumflex; $L$, left; $B A V$, bicuspid aortic valve. 

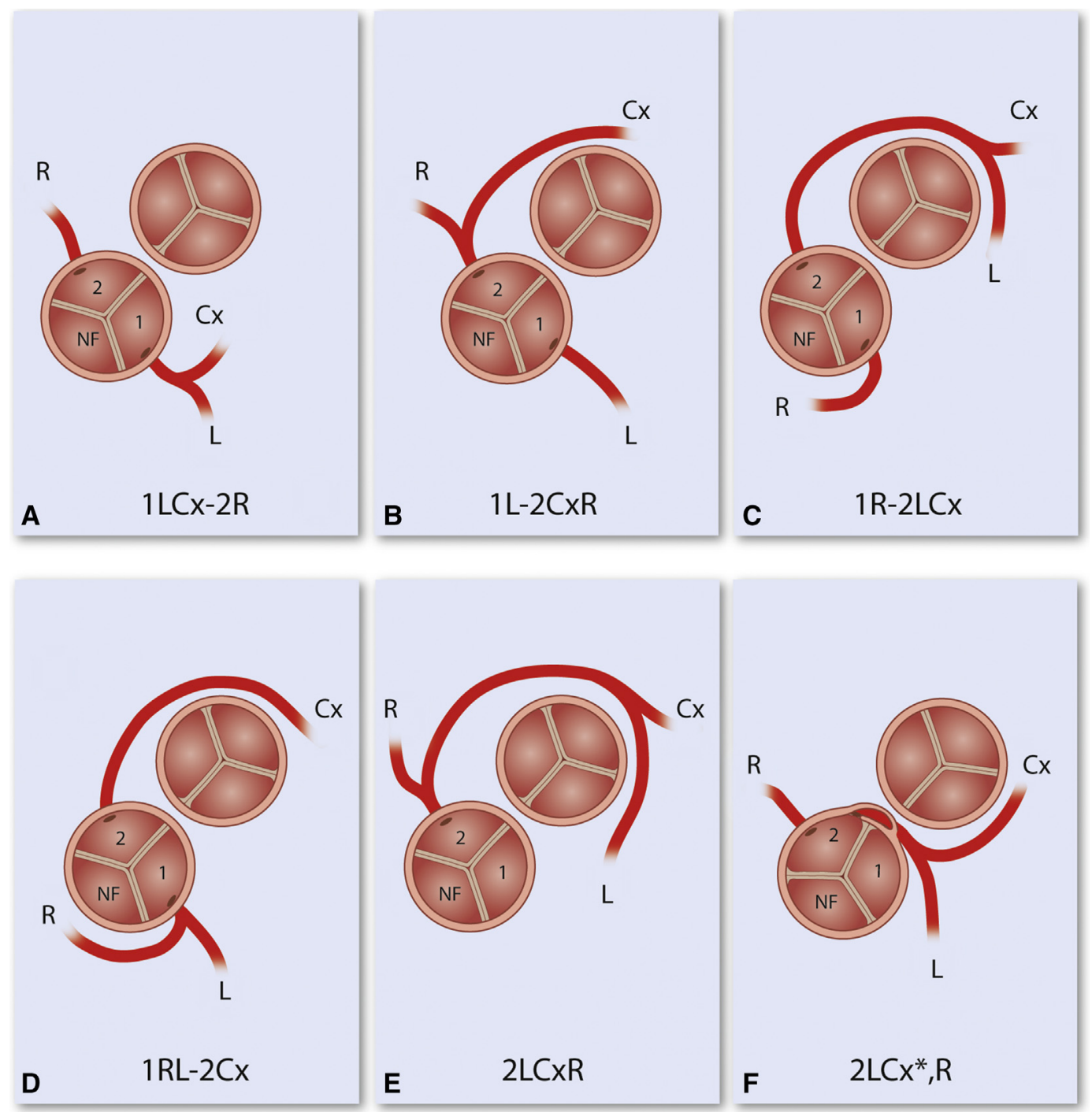

FIGURE 4. Variations in coronary anatomy in TGA, view from above. A, The most common form. B-D, Variations with a double sinus origin. E, Example of a single sinus 2 origin. F, The clinically most hazardous form is shown with the LCx taking off directly behind the facing commissure from sinus 2 and thereafter taking an interarterial/intramural course to the anterior aspect of the heart (indicated by an asterisk in the coding). The combination of $\mathrm{L}$ and $\mathrm{Cx}$ thereafter courses over the right ventricular outflow tract crossing anteriorly to the pulmonary orifice. $R$, Right; $N F$, nonfacing; $C x$, circumflex; $L$, left.

The second step is to simplify the description of the coronary ostium (defined by the position of the visible orifice) and branching patterns of the main coronary arteries. When describing the coronary anatomy according to the Leiden Convention, the sinus number is indicated first (as explained in step 1), and thereafter the coronary branch that arises from sinus 1 is annotated proceeding in a counterclockwise fashion (step 2; Figure $3, B$ ). For a normal heart, this would be $1 \mathrm{R}-2 \mathrm{LCx}$, indicating the 3 main branches being the RCA (R), the LAD (L), and the LCx $(\mathrm{Cx})$ (Figure 3, B). The LCA, composed of the $\mathrm{L}$ and $\mathrm{Cx}$ (with or without a main stem), does not have a separate annotation. To cover variations in coronary orifice origin, step 3 is necessary (Figure 3, C and $D$ ). The use of a dash (-) between artery branches arising from a different sinus is applicable in all cases with double sinus origin (Figure 3, $B$ and $C$ ). When coronary artery branches share a common ostium or stem, there is no comma (Figure $3, B$ ) between the annotations, whereas a comma is interposed when 2 (or more) arteries arise separately from the same sinus (Figure $3, C$ and $D$ ). For instance, in case of normal anatomy with an LCA as a main stem, the annotation is $1 \mathrm{R}-2 \mathrm{LCx}$, as described earlier. In case of an absent main stem (separate origin of $\mathrm{L}$ and $\mathrm{Cx}$ from sinus 2), the annotation would be $1 \mathrm{R}$ 2L,Cx. An asterisk (*) is provided for a coronary artery branch that has an interarterial, often partly intramural, course (Figure 3, D). These cases may clinically be most at risk of complications, such as sudden cardiac death (SCD). Additional features such as a prepulmonary or a retro-aortic course become evident when the system is applied consequently and indicating the course of the coronary artery separately is not necessary.

Finally, additional information (Figure 3,E) regarding associated anomalies and clinically relevant issues, such as an interarterial/intramural coronary artery course, acute angulation, and position/height of coronary orifices within the sinus, should be described. This is particularly important to underline potentially harmful clinical findings, for example, during the conveyance of medical information to the thoracic surgeon and other professionals involved in patient management. 


\section{Coronary Artery Coding in Transposition of the Great Arteries and Double Outlet Right Ventricle}

The proposed simple coding system was originally devised for TGA and the Taussig-Bing malformation when the arterial switch procedure, initiated by Jatene and colleagues, ${ }^{14}$ became the preferred surgical technique, replacing the atrial switch procedures. In the arterial switch procedure, it is necessary not only to switch the main great arteries but also to transfer the coronary arteries to the neoaortic root (former pulmonary root). Coding systems in which positional semilunar sinus terminology has been used, combined with other morphologic characteristics of the coronary orifice origin and branching patterns, could lead to as many as 28 variations. ${ }^{15}$ Because this was too cumbersome for practical clinical use, several more simple classifications were introduced, mostly making use of an alphabetical identification such as type $\mathrm{A}$ to $\mathrm{D}^{16}$ or of a numeric grouping with subgroups. ${ }^{12}$ The original "Leiden Convention," using facing sinus 1 and 2 with an additional numeric identification, left 6 basic types, nevertheless requiring memorizing the numerical indications. ${ }^{4}$

The modified Leiden Convention includes the course of the main branches around the great arteries, resulting in a further redundancy of the number of variations in the coding system. It is fascinating that a rather stable pattern of coronary origin (1LCx-2R) exists in the most common form of TGA with the aortic orifice in a right anterior position (Figure 4, A) as observed in a series of 103 postmortem specimens with TGA (Figure $5, A){ }^{4}$ In case of side-by-side great arteries, as often seen in Taussig-Bing type DORV, the greatest variation in coronary origin like $1 \mathrm{~L}-2 \mathrm{CxR}, 1 \mathrm{R}-2 \mathrm{LCx}$, or 1RL-2Cx (Figures $4, B-D$ and $5, B$ ) is to be expected, ${ }^{4}$ as if the coronary artery has difficulty in finding its shortest course toward a facing semilunar sinus. In case of a frontal position of the aorta this variation is diminished because a shortest course is again clear (Figure 5, C).

It is important that during surgical transfer of the coronary arteries, no kinking occurs, because it may lead to hypoxia of the coronary arterial myocardial perfusion area. Surgical risks are encountered in cases with a single sinus coronary artery pattern. ${ }^{12}$ A surgical challenge is also found in cases with an interarterial/intramural course (Figures 4, F, and 5, A and $B$ ) of the coronary artery between the aortic and pulmonary orifice, requiring an adaptation of the transfer procedure. ${ }^{12,17,18}$ These cases often present with a double orifice from 1 sinus, with the interarterial coronary artery originating from the opposite sinus (eg, an interarterial LCA originating from the right sinus) and coursing directly behind or most often just above the facing commissure of the aortic and pulmonary orifices. In these cases, there is an acute take-off with a slit-like coronary orifice while the first part of the artery takes an intramural course in the wall of the aorta, where both the aorta and the coronary artery have a joined media without a separating adventitia. ${ }^{10,19}$

\section{Coronary Artery Coding Variations in Normally Related Great Arteries}

The estimated incidence of coronary anomalies in structurally normal heart ranges between $0.1 \%$ and $1.0 \%$, although this is difficult to estimate because many variations are likely asymptomatic. ${ }^{10}$ Although the incidence is low, unfavorable anatomy can have a high clinical impact, and coronary anomalies are held responsible for approximately $20 \%$ of cases of SCD in competitive athletes. ${ }^{20} \mathrm{~A}$ large magnetic resonance imaging

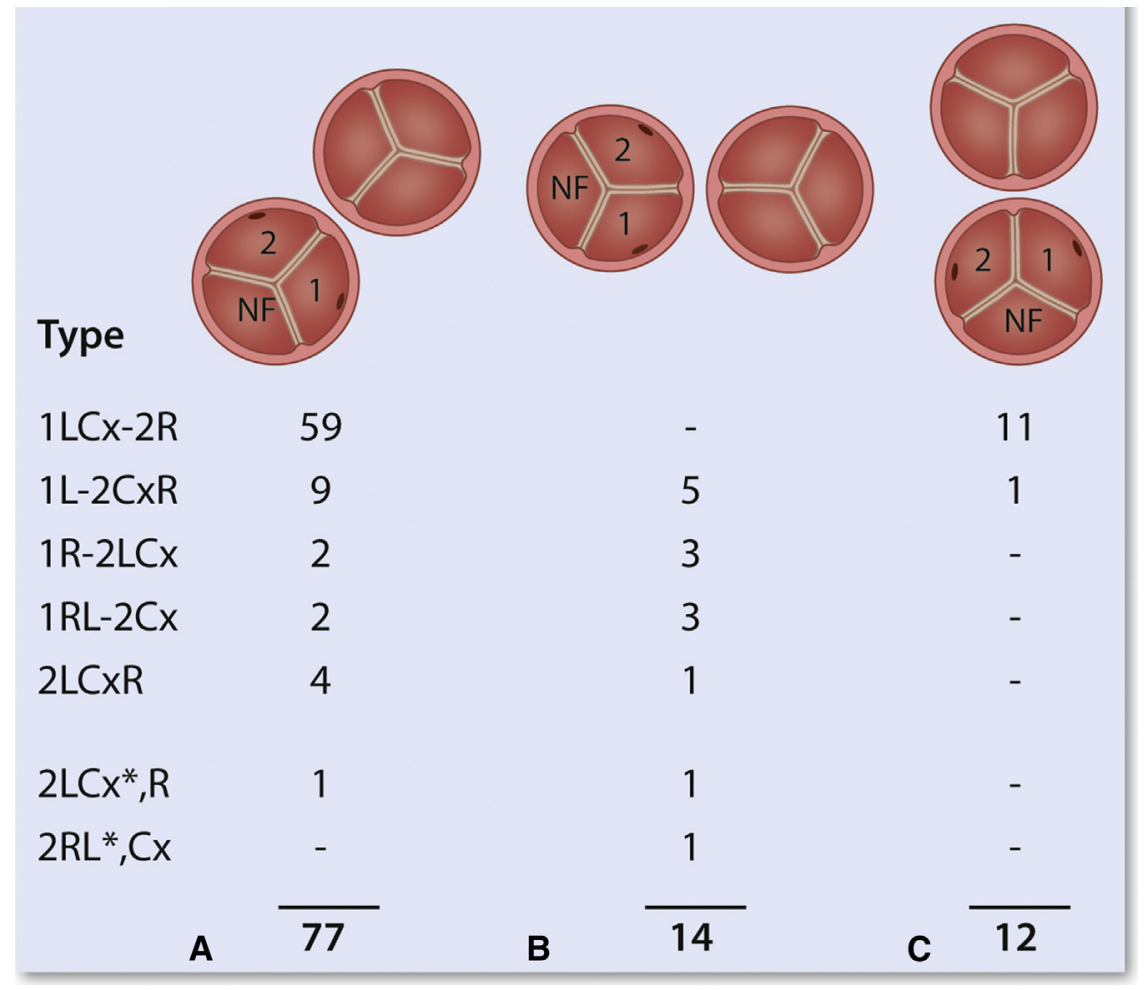

FIGURE 5. Frequency of variations in 103 cases TGA with right anterior position of the aorta (A), side-by-side position of the great arteries in DORV with subpulmonary ventricular septal defect (B), and anterior position of the aorta (C) (modified after Gittenberger-de Groot and colleagues ${ }^{4}$ ). The most common form $(1 \mathrm{LCx}-2 \mathrm{R})$ is found in cases with a right anterior position of the aorta. Of the complicated cases with an interarterial/intramural artery (1LCx*,R), 1 is found in the most common position seen in TGA, being right anterior, and 2 cases ( $1 \mathrm{LCx} *, \mathrm{R}$ and $2 \mathrm{RL} * \mathrm{Cx})$ were seen in side-by-side great artery positions, mostly encountered in DORV. $N F$, Nonfacing. 
screening program found that $0.7 \%$ of the population had an interarterial course. The origin of the RCA from the opposite sinus was 3 to 6 times more common than an aberrant LCA. ${ }^{21}$ The coronary anatomy mostly related to SCD is single sinus coronary anatomy, especially an LCA originating from the right sinus, that is associated with an interarterial/intramural course and acute take-off. ${ }^{10}$ When applying the modified Leiden Convention in cases with normally related great arteries, this results in a set of variations that is exemplified in Figure 6, $A$ to $F$. Figure 6 covers multiple possibilities of the system but is not meant to be complete. As described earlier, the abnormally located ostium of the LCA branch in combination with an intramural course that specifically tends to cross above or behind the facing aortic commissure carries the highest risk of sudden death (Figure 6, F). ${ }^{10}$

\section{Coronary Artery Coding in Bicuspid Aortic Valves}

Classification of coronary artery origin tends to be challenging when not all 3 valvular commissures can be distinguished, as is the case in BAV. BAV has recently gained increased interest because of the often concomitant dilation of the ascending aorta in early adult life. ${ }^{22}$ Surgical treatment strategies focus on the possibility to predict development of aortic dilation based on the types of aortic valve morphology. We have adopted and modified the Schaeffer and colleagues ${ }^{23}$ and Sievers and Schmidtke ${ }^{24}$ numeric classification to 3 types based on which valve leaflets are conjoined and whether or not a raphe can be distinguished. In contrast to the classification by Sievers and Schmidtke, ${ }^{24}$ this adjusted classification does not specifically mention the number of raphes. We propose that a numeric valve annotation can also be used for BAV variations (Figure 7). It is generally
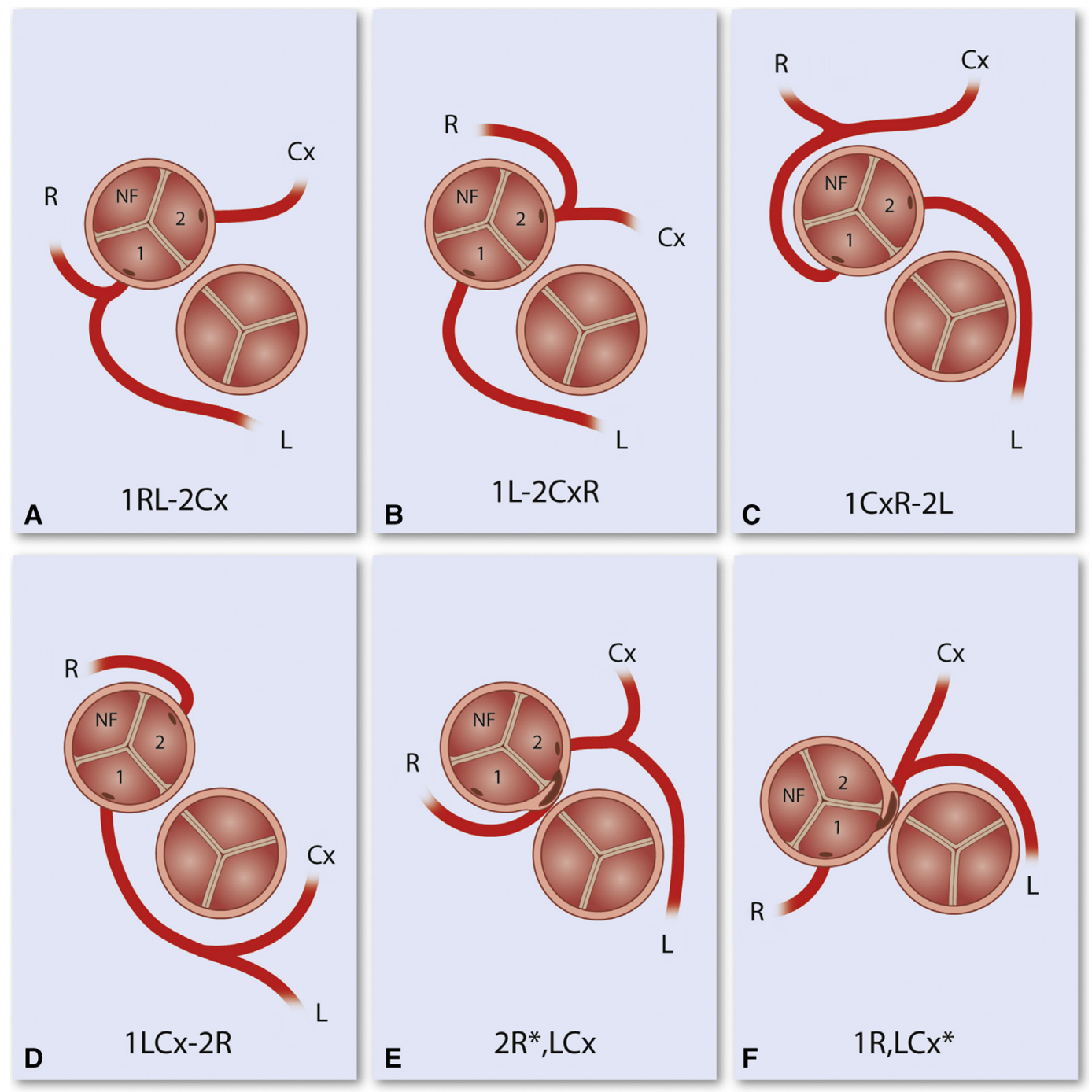

FIGURE 6. This schematic figure presents the most common variations in coronary arterial patterning, viewed from above. A, The normally encountered situation. B-F, Less common variations. A dash (-) is used between branches that derive from a separate sinus (A-D). A comma is interposed when the branches arise from a separate orifice in the sinus (E, F). An asterisk is placed after an intra-arterial/intramural branch (E, F). Variation F is clinically the most hazardous with an interarterial/intramural course of a branch. In these cases, the artery commonly shares part of the aortic wall media with no adventitia interposed. The orifice of the artery is commonly present in the direct neighborhood of the facing commissure. $R$, Right; $N F$, nonfacing; $C x$, circumflex; $L$, left. 
Type 1A

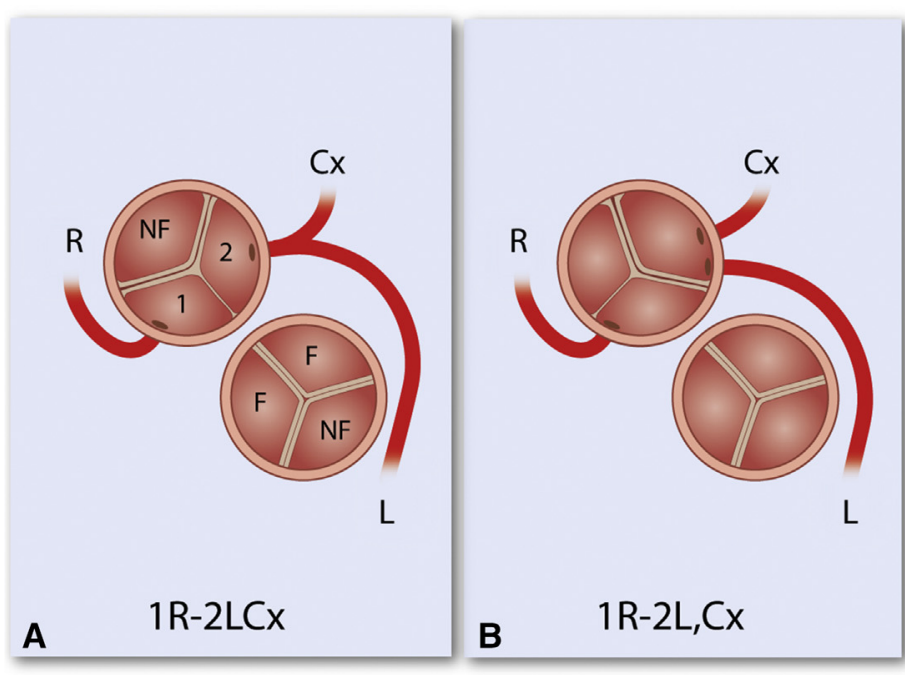

Type 1B

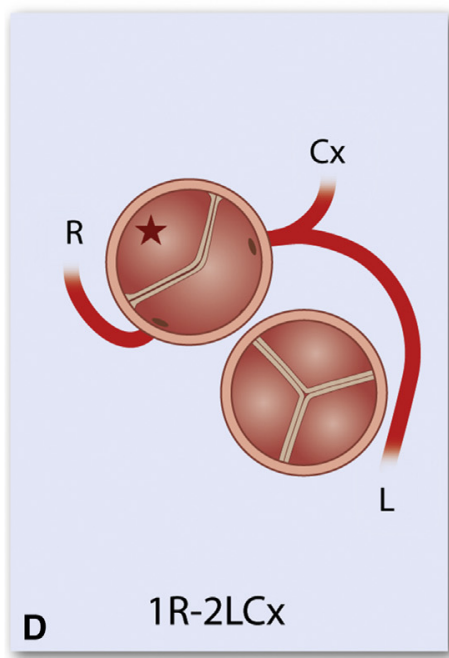

Type $2 \mathrm{~A}$

Type 2B

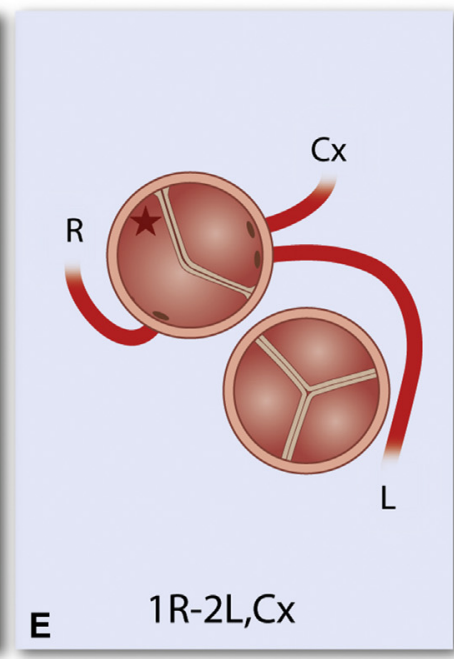

Type $3 \mathrm{~A}$

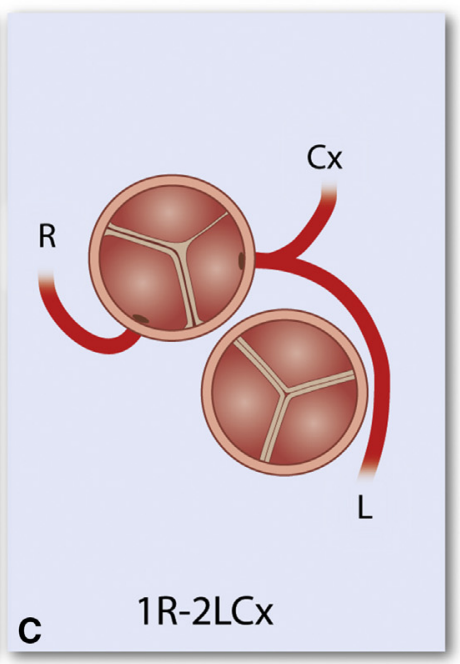

Type 3B

FIGURE 7. Schematic representation of classification in BAV disease with associated variations in coronary anatomy viewed from above (A-F), for example, separate ostia of the left main stem (B, E). The supposed "nonfacing/noncoronary" part of the aortic leaflet in case of a strictly bicuspid valve is indicated by a star in D to F. $R$, right; $N F$, nonfacing; $C x$, Circumflex; $L$, left.

assumed that in BAV, in which only 2 functional leaflets develop, there is a fusion (or nonseparation) of 2 of the 3 leaflets. In the classification used in the current article, type 1 has a joined right (facing sinus 1 ) and left (facing sinus 2 ) semilunar sinus, whereas in type 2 the right (sinus 1 ) and the noncoronary (nonfacing sinus) are joined. Type 3 is rare with a fusion of the left (sinus 2) and the nonfacing sinus (Figure 7). On the basis of the presence of a commissural ridge or raphe or the complete absence of a raphe, the letter A for presence (Figure 7, $A-C$ ) and B for absence (Figure 7, D-F) was assigned.

The modified Leiden Convention coronary coding system can be used in the majority of BAVs with a raphe (type A) based on the general rule that in $99 \%$ of the hearts the coronary artery connects to a facing sinus 1 and 2 and the nonfacing sinus remains noncoronary. A single sinus coronary origin has been reported to be rare in patients with BAV. ${ }^{25}$ The most common varieties observed in BAV are a left dominant coronary anatomy ${ }^{26}$ and absence of a short left main stem (Figure 7, $B$ and $E$ ), which may complicate cardioplegia when this is not recognized. Coronary coding is challenging in type B BAV (without raphe). Here, reasoning could be reversed and the modified Leiden Convention could be used to predict which leaflets have been fused or not separated during development and thus could be decided whether it is a type 1-, 2-, or 3related morphology. The normal (tricuspid) aortic valve is composed of 2 "facing" leaflets and a facing commissure (facing the corresponding pulmonary valve leaflets and commissure) and 1 "nonfacing" leaflet (Figure 2, $C$ and $D$ ). In case of type B BAV, we know that the most common form is type $1 \mathrm{~B} .{ }^{26}$ If this is the case, the "potential raphe" between the coronary branches should point toward the facing commissure of the pulmonary semilunar valve (Figure 7, $A$ and $D$ ). In case of type $2 \mathrm{~B}$ and 
3B, 1 of the commissures of the BAV aortic valve points toward the facing commissure of the pulmonary valve, thus allowing distinction between type $1 \mathrm{~B}$ and types 2 and $3 \mathrm{~B}$. It is more difficult to distinguish between a type $2 \mathrm{~B}$ and $3 \mathrm{~B}$. In this case, the clinically observed opening pattern of the aortic valve should be assessed. After identification of the (potential) nonfacing/noncoronary leaflet (red stars in Figure 7, D-F), the Leiden Convention nomenclature can be applied. If determination of valve morphology is inconclusive, a descriptive approach is recommended. This should be added to the report of associated characteristics (Figure 3,E). Data regarding coronary arterial patterning and origin in $\mathrm{BAV}$ as related to BAV subtypes are scarce. Recently, our group published data showing variations in left-right dominance ${ }^{26}$ and an increased incidence of high take-off coronary arteries in BAV, compared with CHD in general. ${ }^{27}$ This also belongs to the additional information that needs to be provided to the modified Leiden Convention coding system (Figure 3, E).

\section{Associated Characteristics With Respect to Coronary Orifices, Course of Coronary Arterial Branches, and Anatomic Variations}

In some cases, it is necessary to describe a number of essential aspects of coronary variations that may have an impact on the surgical treatment of choice.

These include the following (Figure 3,E):

1. The presence of an intramural course in cases with an interarterial branch, which is especially hazardous if it concerns the LCA branches.

2. The relative relationship of the great arterial orifices (Figure 1).

3. The eccentric positioning of an orifice in the horizontal plane is of importance for the possibility to excise a proper button. Positioning close to a valvular commissure and acute angle take-off are features that hamper the procedure.

4. The degree of alignment of the facing commissures to determine the site of repositioning the buttons of the new coronary orifice in an arterial switch procedure.

5. The positioning of an orifice in a vertical plane above the sinotubular junction, referred to as "high take-off," often seen in concordance with an acute angle take-off and a variable intramural course. This angle is also influenced by aortic diameter; an acute angle can become more outspoken with aortic dilation. ${ }^{28}$

6. The presence of a single, double, or common orifice for branches is an essential part of preoperative knowledge.

7. Accessory branches that bud off the coronary sinuses should be indicated, as well as the origin of the sinoatrial node branch and a possible conal artery.

8. Information on right, left, or co-dominance of the coronary artery system.

\section{CONCLUSIONS}

Coronary arterial patterning is of relevance, especially in the setting of CHD in which preoperative information before the often complex surgery is mandatory. TGA and DORV with subpulmonary ventricular septal defect require special adjustments for the transfer of the coronary arteries to the neoaorta, specifically in case of an interarterial/intramural course. In normally related great arteries, the interarterial/intramural course of specifically the LCA branches poses a life-threatening hazard that can be fatal in young adults during athletic performances. ${ }^{9,10} \mathrm{We}$ propose an accessible unambiguous system based on the modification of a previously developed and widely implemented coding system referred to as the "Leiden Convention," extending its use for several forms of CHD and including information on origin and branching patterns.

\section{Conflict of Interest Statement}

Authors have nothing to disclose with regard to commercial support.

\section{References}

1. Angelini P. Normal and anomalous coronary arteries: definitions and classification. Am Heart J. 1989;117:418-34.

2. Chiu IS, Anderson RH. Can we better understand the known variations in coronary arterial anatomy? Ann Thorac Surg. 2012;94:1751-60.

3. Weaver ME, Pantely GA, Bristow JD, Ladley HD. A quantitative study of the anatomy and distribution of coronary arteries in swine in comparison with other animals and man. Cardiovasc Res. 1986;20:907-17.

4. Gittenberger-de Groot AC, Sauer U, Oppenheimer-Dekker A, Quaegebeur J. Coronary arterial anatomy in transposition of the great arteries: a morphologic study. Pediatr Cardiol. 1983;4:15-24.

5. Bogers AJ, Gittenberger-de Groot AC, Poelmann RE, Peault BM, Huysmans HA. Development of the origin of the coronary arteries, a matter of ingrowth or outgrowth? Anat Embryol (Berl). 1989;180:437-41.

6. Tian X, Hu T, Zhang H, He L, Huang X, Liu Q, et al. Subepicardial endothelial cells invade the embryonic ventricle wall to form coronary arteries. Cell Res. 2013;23:1075-90.

7. Theveniau-Ruissy M, Perez-Pomares JM, Parisot P, Baldini A, Miquerol L, Kelly RG. Coronary stem development in wild-type and Tbx 1 null mouse hearts. Dev Dyn. 2016;245:445-59.

8. Lacour-Gayet F, Anderson RH. A uniform surgical technique for transfer of both simple and complex patterns of the coronary arteries during the arterial switch procedure. Cardiol Young. 2005;15(Suppl 1):93-101.

9. Basso C, Corrado D, Thiene G. Congenital coronary artery anomalies as an important cause of sudden death in the young. Cardiol Rev. 2001;9: 312-7.

10. Brothers JA, Frommelt MA, Jaquiss RDB, Myerburg RJ, Fraser CD Jr, Tweddell JS. Expert consensus guidelines: anomalous aortic origin of a coronary artery. J Thorac Cardiovasc Surg. 2017;153:1440-57.

11. Kooij M, Vliegen HW, de Graaf MA, Hazekamp MG. Surgical treatment of aberrant aortic origin of coronary arteries. Eur J Cardiothorac Surg. 2015;48:724-30.

12. Suzuki T. Modification of the arterial switch operation for transposition of the great arteries with complex coronary artery patterns. Gen Thorac Cardiovasc Surg. 2009;57:281-92.

13. Quaegebeur J. The Arterial Switch Operation-Rationale, Results, Perspectives [thesis/dissertation]. Leiden, The Netherlands: Leiden University; 1986.

14. Jatene AD, Fontes VF, Paulista PP, Souza LC, Neger F, Galantier M, et al. Anatomic correction of transposition of the great vessels. J Thorac Cardiovasc Surg. 1976;72:364-70.

15. Shaher RM, Puddu GC. Coronary arterial anatomy in complete transposition of the great vessels. Am J Cardiol. 1966;17:355-61.

16. Yacoub MH, Radley-Smith R. Anatomy of the coronary arteries in transposition of the great arteries and methods for their transfer in anatomical correction. Tho$\operatorname{rax}$ 1978;33:418-24.

17. Kaneko Y, Tsuchiya K, Yamamoto Y, Yoda H, Yamamoto W, Kobayashi J. Arterial switch in a 1146-gram neonate with transposition of the great arteries and an intramural coronary artery. J Thorac Cardiovasc Surg. 2007;134:1064-5.

18. Koshiyama H, Nagashima M, Matsumura G, Hiramatsu T, Nakanishi T, Yamazaki K. Arterial switch operation with and without coronary relocation for intramural coronary arteries. Ann Thorac Surg. 2016;102:1353-9.

19. Gittenberger-de Groot AC, Sauer U, Quaegebeur J. Aortic intramural coronary artery in three hearts with transposition of the great arteries. J Thorac Cardiovasc Surg. 1986;91:566-71.

20. Maron BJ, Thompson PD, Puffer JC, McGrew CA, Strong WB, Douglas PS, et al. Cardiovascular preparticipation screening of competitive athletes. A statement for health professionals from the sudden death committee (clinical cardiology) and congenital cardiac defects committee (cardiovascular disease in the young), American Heart Association. Circulation. 1996;94:850-6. 
21. Angelini P. Novel imaging of coronary artery anomalies to assess their prevalence, the causes of clinical symptoms, and the risk of sudden cardiac death. Circ Cardiovasc Imaging. 2014;7:747-54.

22. Grewal N, Gittenberger-de Groot AC, Poelmann RE, Klautz RJ, Lindeman JH, Goumans MJ, et al. Ascending aorta dilation in association with bicuspid aortic valve: a maturation defect of the aortic wall. J Thorac Cardiovasc Surg. 2014; 148:1583-90

23. Schaefer BM, Lewin MB, Stout KK, Gill E, Prueitt A, Byers PH, et al. The bicuspid aortic valve: an integrated phenotypic classification of leaflet morphology and aortic root shape. Heart. 2008;94:1634-8.

24. Sievers HH, Schmidtke C. A classification system for the bicuspid aortic valve from 304 surgical specimens. J Thorac Cardiovasc Surg. 2007;133: 1226-33.

25. Rashid A, Saucedo JF, Hennebry TA. Association of single coronary artery and congenital bicuspid aortic valve with review of literature. J Interv Cardiol. 2005; 18:389-91.
26. Koenraadt WM, Tokmaji G, DeRuiter MC, Vliegen HW, Scholte AJ Siebelink HM, et al. Coronary anatomy as related to bicuspid aortic valve morphology. Heart. 2016;102:943-9.

27. Koenraadt WMC, Bartelings MM, Bokenkamp R, Gittenberger-de Groot AC DeRuiter MC, Schalij MJ, et al. Coronary anatomy in children with bicuspid aortic valves and associated congenital heart disease. Heart. 2018;104:385-93.

28. Veltman CE, Beeres SL, Kalkman DN, Kelder TP, Kies P, Vliegen HW, et al. Variation in coronary anatomy in adult patients late after arterial switch operation: a computed tomography coronary angiography study. Ann Thorac Surg. 2013;96:1390-7.

Key Words: bicuspid aortic valve, double outlet right ventricle, embryonic development, transposition of the great arteries, ventricular septal defect 